\title{
Research on Ultrasonic Testing Technology of Bearing Bush of Turbo Generator
}

\author{
Jian Yin, Yuheng Ning \\ Datang Northeast Electric Power Test \& Research Institute, Changchun, China
}

Keywords: bearing bush, ultrasound, detector, sensitivity, defect judgment, dovetail

\begin{abstract}
Various problems of different levels in the ultrasonic testing standards of bearing bush of power station take place at present, which has certain influence on the actual defect judgment. According to the theoretical analysis and practical test results, the technology in this paper has the advantages as follows. First, the defining standards of the frequency and size of the probe used in the test are provided. Second, the adjustment method of sensitivity detection is simple and practical, which can effectively detect the delamination defects. Third, after the clearing up of the defect judgment methods, the paper recommends using the Technology Conditions of Bearing Bush of Turbine Babbitt Metal (JB/T4272-1994, equalling to ISO4386-1:1992) and typical layered waveform provided in the paper to judge the defects. Fourth, the paper provides the quantitative judgement method of defects according to the Nondestructive Testing of Pressure EquipmentsPart 3: Ultrasonic Testing (JB/T4730-2005) and specification for MHI bearing bush testing for quantitative analysis of defects. Fifth, the specific testing methods of bearing bush of turbo generator with dovetail are provided in the paper.
\end{abstract}

\section{Introduction to Testing Standards of Bearing Bush}

At present, the ultrasonic testing standards of bearing bush of turbo generator in power stations are carried out according to Technology conditions of bearing bush of Tin-based alloy of turbo generator (JB/T 4272-1994) and The ultrasonic testing for alloy bearing of in-service turbogenerator (DL/T297-2011) in China. There are still many problems in the execution process of the two standards, which has certain influence on the actual defect judgment.

The problems in the execution process of the Technology conditions of bearing bush of Tinbased alloy of turbo generator (JB/T 4272-1994, equalling to ISO4386-1: 1992) are shown as follows:

(1) The standards recommend the double crystal probe for testing. However, due to the existence of focus, the sensitivity of focus area by double crystal focusing probes is high and the operational is insufficient. At the same time, as a result of irregularity of reflected wave of double crystal focusing probes, the defect judgement by reflected wave's amplitude can be made only once, which can bring error in practical applications.

(2) The specific stipulations of scanning the sensitivity are absent in the standards. The standards judge the delamination defects only according to the bottom-echo amplitude, not considering the amplitude of the interface wave.

(3) The specific stipulations of standard test blocks are absent in the standards. The specific regulations of artificial defect size and the dimension type are not provided in the standards.

(4) The quantitative stipulations are absent in the standards and the defect areas cannot be confirmed.

The problems in the execution process of The ultrasonic testing for alloy bearing of in-service turbo-generator (DL/T297-2011) are shown as follows:

(1) The standards recommend the double crystal square probe for testing. In the practical testing process, the square probe is liable to scratch the interface of Babbit metal. At the same time, the square probe is against to the coupling of workpiece interface.

(2) The standards require using the reference block method to adjust the testing sensitivity in the testing process. The testing blocks of different categories should be made to adapt to the alloy 
bushes of different thickness, which is not strong at operability.

(3) The quantitative requirements of the defects are not clear in the standards. The methods to define the defect boundaries and the categories of waveform are not provided in the standards.

After referring the testing methods of bearing bush in foreign countries, the author will analyse the bearing bush testing technology from the aspects of probe selection, sensitivity adjustment, defects judgement, defects quantification to provide a new direction of bearing bush testing in China.

\section{Technology Analysis of Bearing Bush Testing}

\subsection{Selection of Probes}

For the ultrasonic testing methods, the selection of a suitable probe is a prerequisite for the development of technology. Table 1 is about the requirements of the probe frequency and the chip size of alloy bushing of the testing standards at home and abroad. As can be seen in Table 1, the alloy surface probes of bearing bush testing can be commonly categorized into two main areas: one crystal probe and double crystal probe. The common frequency is $5 \mathrm{MHz}$ and the size of the chip is generally controlled within $10 \mathrm{~mm}$.

Table 1. Probes selection under the different standards

\begin{tabular}{|c|c|c|c|}
\hline \multirow{2}{*}{ Applicable standards } & $\begin{array}{c}\text { Probe } \\
\text { classification }\end{array}$ & $\begin{array}{c}\text { Probe } \\
\text { frequency } \\
\text { /MHZ }\end{array}$ & Chip siez/mm \\
\hline \multirow{2}{*}{ DL/T 297-2011 } & one crystal & $2-5$ & $\Phi 4-8$ \\
\cline { 2 - 4 } & double crystal & 5 & $2 * 4 \times 4$ \\
\cline { 2 - 4 } & & & $2 * 10 \times 10$ \\
\hline JB/T4272-1994 (equaling to ISO4386-1:1992) & one crystal & $2-5$ & $\Phi 10-30$ \\
\hline \multirow{2}{*}{ MHI rules } & double crystal & 5 & $\Phi 10-15$ \\
\hline ASTM B23(SA578) & one crystal & 5 & $\Phi 5-10$ \\
\hline
\end{tabular}

For the ultrasonic testing of the turbine bearing bush, it is recommended to use the single crystal probe with high frequency and small size under the condition that the height of the bottom reflection wave is ensured (not less than $80 \%$ of the full screen amplitude). The reasons are as follows:

(1) The reason for not choosing the double crystal probe is clarified in the previous. We won't explore it in again this article.

(2) The selection of probes with smaller diameters can decrease the near-field and increase the contact area of the probe and the testing surface of bearing bush. Therefore, under the condition of ensuing penetrating power, we will give priority to the probes with smaller diameters.

(3) With the current manufacturing level of the probe, the blind area of the single crystal probe can reach $2 \mathrm{~mm}$, and the blind area of some of the imported probes can even reach $0.5 \mathrm{~mm}$. The thickness of Babbitt metal layer of bearing bush of turbine generator generally ranges from $5 \mathrm{~mm}$ to $12 \mathrm{~mm}$. The single crystal probe has met the testing requirements. It is another reason that the straight probe can replace the double crystal probe.

(4) The advantages of high efficiency of probe lie in stronger emitted energy of ultrasonic wave, better directivity of sound waves, fewer areas of blind regions and higher resolution of little defect.

(5) When the thickness of Babbitt metal layer of bearing bush of turbine generator is more than $8 \mathrm{~mm}$, the probe with high frequency is conductive to the defect diagnose.

The reflective amplitudes of the primary wave and the secondary wave in the interface are shown in Table 2. As seen from the Table 2, the application of the low-frequency probe can raise the 
amplitude of the secondary wave in the interface, which can influence on the defect judgement. Therefore, in the case of penetrating power assurance, we should decrease the amplitude of the secondary wave in the interface as far as possible. To summarize, we recommend the 5PФ8 single crystal probe in the ultrasonic testing of bearing bush of turbo generator.

Table 2. Reflective amplitudes of the primary wave and the secondary wave in the interface of different frequency probes (actual testing value)

\begin{tabular}{|c|c|c|c|}
\hline \multirow{2}{*}{$\begin{array}{c}\text { Specifications of } \\
\text { probe/MHZ,ømm }\end{array}$} & $\begin{array}{c}|c| \\
\text { Primary reflection } \\
\text { of alloy interface }\end{array}$ & $\begin{array}{c}\text { Conditions of actual testing } \\
\text { Primary reflective amplitude } \\
\text { under the condition of good } \\
\text { combination layer of alloy \% }\end{array}$ & $\begin{array}{c}\text { Secondary reflective } \\
\text { amplitude } 10 \%\end{array}$ \\
\cline { 2 - 4 } & $\begin{array}{c}\text { db value of } 80 \% \\
\text { amplitude/dB }\end{array}$ & 80 & 10 \\
\hline $2.5 / 8$ & 52.5 & 80 & 8 \\
\hline $2.5 / 14$ & 58 & 80 & 6 \\
\hline $4 / 8$ & 55 & 80 & 6 \\
\hline $5 / 8$ & 80.5 & & \\
\hline
\end{tabular}

\subsection{Adjustment of Sensitivity}

The specifications of MHI bearing bush testing set out the ultrasonic testing can be applied by the way of Babbit metal block or carbon steel block to adjust the testing sensitivity. According to the blocks, the detection sensitivity can be adjusted in any way in Table 3.

Table 3. Adjustment method of sensitivity tested by ultrasonic wave

\begin{tabular}{|c|c|c|c|}
\hline Block name & $\begin{array}{c}\text { Block } \\
\text { thickness/mm }\end{array}$ & detection sensitivity of 5Z10N & detection sensitivity of 5Z5N \\
\hline $\begin{array}{c}\text { Bidery metal } \\
\text { TP }\end{array}$ & 8 & B4 $: 100 \%$ & B4 $: 100 \%$ \\
\hline JIS STB-A3 & 16 & B6 $: 100 \%$ & B3 $: 100 \%$ \\
\hline JIS-STB-A1 & 25 & B4 $: 80 \%+6 \mathrm{~dB}$ & B2 $: 100 \%$ \\
\hline
\end{tabular}

Take the adjustment of detection sensitivity of $5 Z 10 \mathrm{~N}$ as an example. The result is shown in Figure 1.

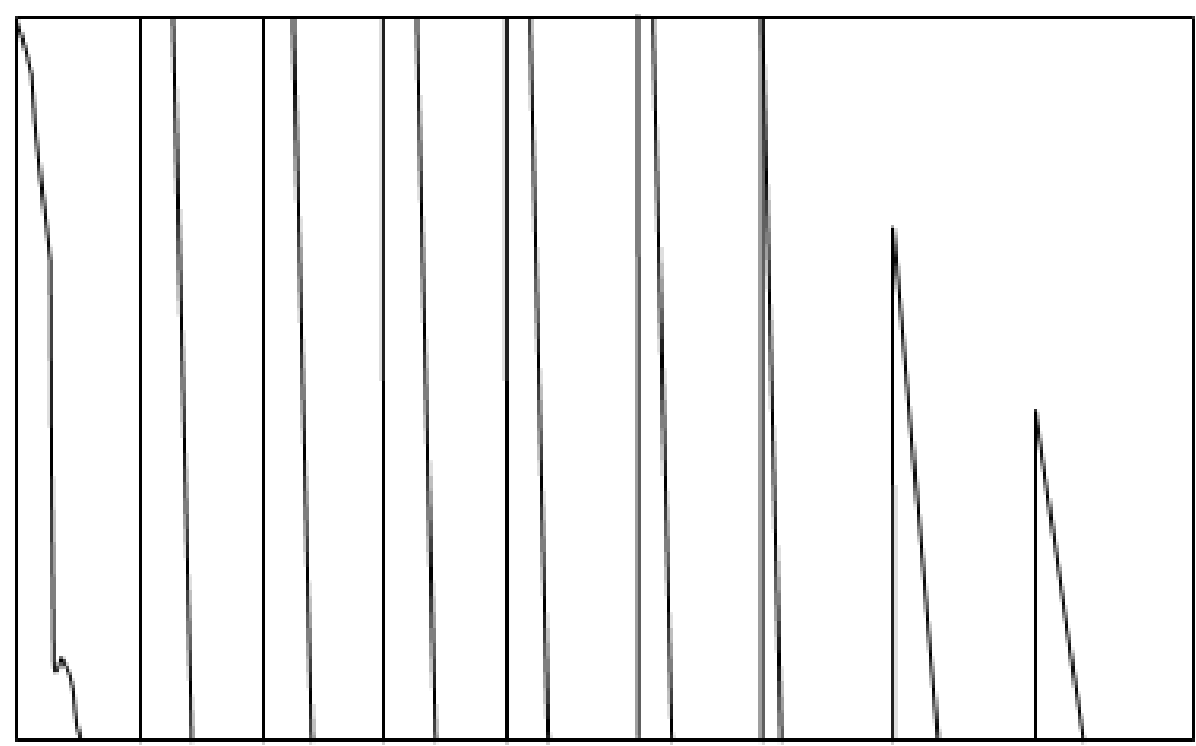

Figure 1. Diagram of detection sensitivity of carbon steel block 
The MHI regulations and other regulations set out the comparison of detection sensitivity, which is shown in Table 4.

Table 4. Adjustment method of sensitivity tested by ultrasonic wave

\begin{tabular}{|l|l|}
\hline \multicolumn{1}{|c|}{ Regulations } & \multicolumn{1}{|c|}{ Rules of detection sensitivity } \\
\hline $\begin{array}{l}\text { The ultrasonic testing for alloy bearing } \\
\text { of } \begin{array}{l}\text { in-service turbo-generator } \\
\text { (DL/T297-2011) }\end{array}\end{array}$ & $\begin{array}{l}\text { The equivalent of } 80 \% \text { of reflective wave of the } \\
\text { interface of bearing bush alloy + (4-6) d }\end{array}$ \\
\hline $\begin{array}{l}\text { Technology Conditions of Bearing Bush } \\
\text { of Turbine Babbitt Metal (equaling to } \\
\text { ISO4386-1:1992) }\end{array}$ & $\begin{array}{l}\text { The equivalent of } 80 \% \text { of reflective wave of the } \\
\text { interface of bearing bush alloy-11dB }\end{array}$ \\
\hline $\begin{array}{l}\text { SA578 (2014) Testing of Special Steel } \\
\text { plate and clad steel plate using } \\
\text { Ultrasonic direct wave }\end{array}$ & $\begin{array}{l}\text { The equivalent of } 80 \% \text { of reflective wave of the } \\
\text { interface of bearing bush alloy -(11-14)dB }\end{array}$ \\
\hline $\begin{array}{l}\text { Testing Regulations of Bearing Bush of } \\
\text { MHI }\end{array}$ & $\begin{array}{l}\text { The equivalent of } 80 \% \text { of reflective wave of the } \\
\text { interface of bearing bush alloy +11dB }\end{array}$ \\
\hline
\end{tabular}

As can be seen from the above table, the detection sensitivity method provided by MHI is not lower than the sensitivity tested by other standards, and the method can be used to adjust the sensitivity of carbon steel block. We recommend the use of MHI in the actual test due to its simple operation.

\section{Judgement of Bearing Bush Defect}

The ultrasonic testing of the bearing bush is mainly to detect the delamination defect of Babbitt and substrate. Some rules are obtained by theoretical calculation in order to determine the waveform amplitude.

\subsection{DB difference between Reflected Wave and Bearing Alloy under the Condition of Good Combination Layer of Alloy}

Under the condition of good combination of bearing alloy layer and basic materials, the reflectivity of sound pressure in the interface is 0.32 . Under the condition of delamination of bearing alloy layer and basic materials, the reflectivity of sound pressure in the interface is approximately equal to 1 , not considering the attenuation and spread.

\subsection{DB difference between Bottom Echo Wave of Basic Material and Interfacial Wave}

It is assumed that the bottom surface of the bearing bush and the interface of the alloy are parallel structures. The difference between the amplitude of bottom echo wave of the bearing bush and the interfacial wave of alloy under the condition of good combination can be calculated by the reciprocating transmission rate of the ultrasonic wave. hen the detection sensitivity is high enough, as a result of the existence of the heterogeneous interface, the interface quality can be judged by the change of the reflection wave of the interface and the amplitude of the bottom wave of basic material.

\subsection{Waveform Judgment of Delamination Defects of Bearing Bush}

Technology Conditions of Bearing Bush of Turbine Babbitt Metal (JB/T4272-1994, equalling to ISO4386-1:1992) is shown in Figure 2 and Figure 3. We don't recommend the waveform due to the disadvantage of double crystal prone.

The wave in the appendix $\mathrm{C}$ of The ultrasonic testing for alloy bearing of in-service turbogenerator (DL/T297-2011) doesn't provide defect judgment combined with the bottom echo wave. The method is liable to bring errors. We also don't recommend. 
The Testing of Special Steel plate and clad steel plate using Ultrasonic direct wave (ASME/SA578-2014) and MHI standards provide only the text description of defect wave with no waveform.
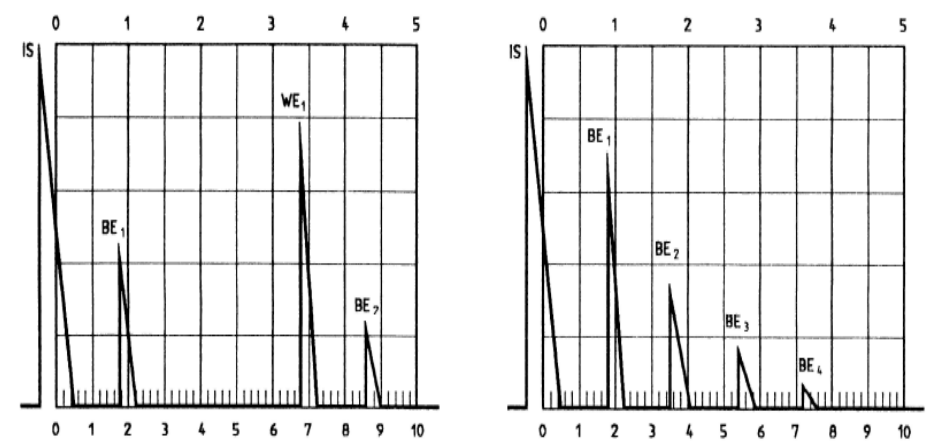

Figure 2. (a): Good interface (b): delamination layer occurs
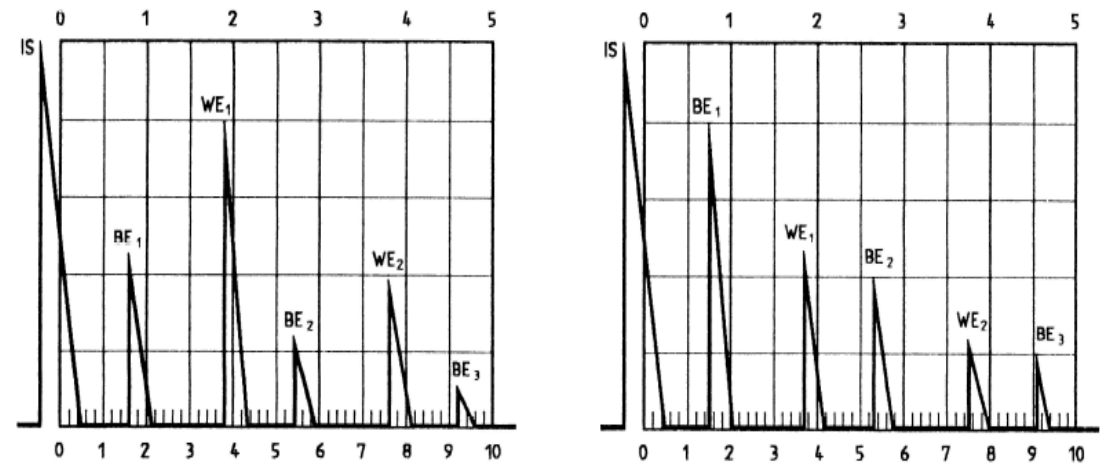

Figure 3. (a): Good interface (b): Bad combination

\section{Quantitative Calculation of Defect Area of Bearing Bush Delamination Layer}

Table 5 shows the near-fields of probes of different categories.

Table 5. near-fields of probes of different categories.

\begin{tabular}{cc}
\hline Probe types/MHZ,ømm & Near-field/mm \\
\hline $2.5 \mathrm{P} \Phi 8$ & 12 \\
\hline $4 \mathrm{P} \Phi 8$ & 19 \\
\hline 5РФ8 & 24 \\
\hline $10 \mathrm{P} \Phi 8$ & 49
\end{tabular}

As we can see from the Table 5, when the thickness of Babbit metal of bearing bush is less than $8 \mathrm{~mm}$, even the probe of 2.5PФ8 cannot avoid the influence of near-field.

Technology Conditions of Bearing Bush of Turbine Babbitt Metal (JB/T4272-1994, equalling to ISO4386-1:1992), Testing of Special Steel plate and clad steel plate using Ultrasonic direct wave (ASME/SA578-2014) and The ultrasonic testing for alloy bearing of in-service turbo-generator (DL/T297-2011) cannot calculate the defect quantitatively. Testing Regulations of Bearing Bush of $M H I$ can calculate the defect quantitatively using the interfacial secondary wave.

\section{Defect Judgement of Edge Region of Dovetail by Ultrasonic Testing}

As is shown in Figure 4, the dovetail groove structure is available in the bearing bush of turbo generator of $600 \mathrm{MW}$ made in China.

The delamination defects of the basic material interface and the Babbit metal in the bottom of 
dovetail cannot be found (blind region). However, the defects in the slope area of dovetail (Region 1 in Figure 5) can be found. When the second-reflected wave is higher than $50 \%$, it can be determined that it is a delamination defect of alloy layer without the appearance of the third wave.
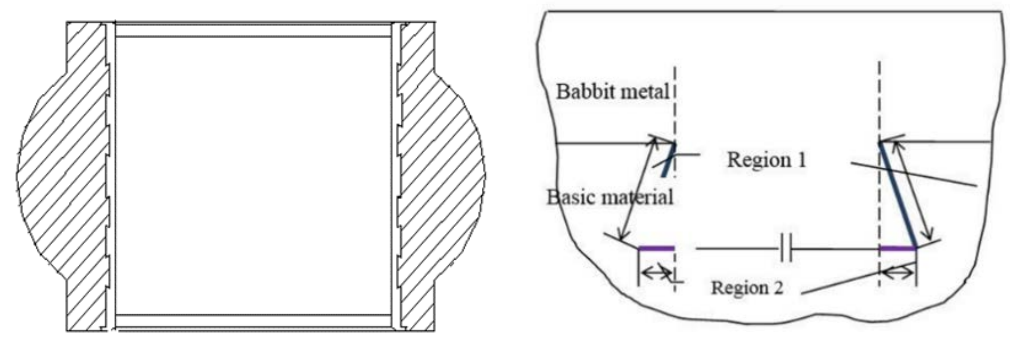

Figure 4. Bearing bush with dovetail

Figure 5. Defected area of the edge region of dovetail of bearing bush

\section{Conclusions}

Various problems of different levels in the ultrasonic testing standards of bearing bush of power station take place at present, which has certain influence on the actual defect judgment. The technology in this paper has the advantages as follows. (1) According to the theoretical analysis and the actual testing results, the defining standards of the frequency and size of the probe are provided. (2) The adjustment method of sensitivity detection is simple and practical, which can effectively detect the delamination defects. (3) After the clearing up of the defect judgment methods, the paper recommends using the Technology Conditions of Bearing Bush of Turbine Babbitt Metal (JB/T4272-1994, equalling to ISO4386-1:1992) and typical layered waveform provided in the paper to judge the defects. (4) The paper provides the quantitative judgement method of defects. According to the Nondestructive Testing of Pressure Equipments-Part 3: Ultrasonic Testing (JB/T4730-2005) and specification for MHI bearing bush testing for quantitative analysis of defects. (5) The paper provides a specific testing method for the bearing bush of turbo generator with dovetail. (6) In the applications process of the above bearing bush testing technology, we successfully found the delamination defects of bearing bush for three times. We accurately determined the defect edge of the delamination layer, which proved the effectivity of the technology.

\section{References}

[1] Li H, Quan Q, Deng Z, et al. A novel noncontact ultrasonic levitating bearing excited by piezoelectric ceramics[J]. Applied Sciences, 2016, 6(10): 280.

[2] Zhao S, Mojrzisch S, Wallaschek J. An ultrasonic levitation journal bearing able to control spindle center position[J]. Mechanical Systems and Signal Processing, 2013, 36(1): 168-181.

[3] Chen X, Liu D, Xu G, et al. Application of wavelet packet entropy flow manifold learning in bearing factory inspection using the ultrasonic technique[J]. Sensors, 2014, 15(1): 341-351.

[4] Schirru M M, Mills R S, Smith O, et al. In situ Measurement of Journal Bearing Lubricant Viscosity by Means of a Novel Ultrasonic Measurement Technique Using Matching Layer[J]. Tribology Transactions, 2017: 1-11.

[5] Dalia S. Abdelhamid, Yingyue Zhang, Daniel R. Lewis, Prabhas V. Moghe, William J. Welsh, and Kathryn E. Uhrich, Tartaric Acid-based Amphiphilic Macromolecules with Ether Linkages Exhibit Enhanced Repression of Oxidized Low Density Lipoprotein Uptake[J]. Biomaterials, 2015(53): 32-39.

[6] Yingyue Zhang, Jennifer W. Chan, Alysha Moretti, and Kathryn E. Uhrich, Designing Polymers with Sugar-based Advantages for Bioactive Delivery Applications [J]. Journal of Controlled Release, 2015(219): 355-368. 
[7] Ishii T, Yamawaki H, Ohuchi H. An Ultrasonic Motor using Thrust Bearing with Dimple Structure on the Friction Surface[J]. Key Engineering Materials, 2015, 649.

[8] Schirru M M, Dwyer-Joyce R S. A model for the reflection of shear ultrasonic waves at a thin liquid film and its application to viscometry in a journal bearing[J]. Proceedings of the Institution of Mechanical Engineers, Part J: Journal of Engineering Tribology, 2016, 230(6): 667-679.

[9] Snoeker B A M, Lindeboom R, Zwinderman A H, et al. Detecting Meniscal Tears in Primary Care: Reproducibility and Accuracy of 2 Weight-Bearing Tests and 1 Non-Weight-Bearing Test[J]. journal of orthopaedic \& sports physical therapy, 2015, 45(9): 693-702. 